\title{
Post-stroke aphasia at the time of COVID-19 pandemic: a telerehabilitation perspective
}

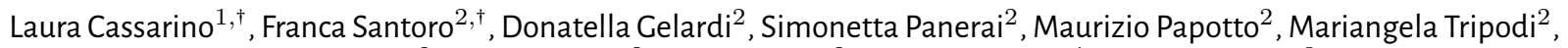
Filomena Irene Ilaria Cosentino ${ }^{2}$, Vincenzo Neri ${ }^{2}$, Raffaele Ferri ${ }^{2}$, Salvatore Ferlito ${ }^{1}$, Daniela Modica ${ }^{3}$, Francesco Fisicaro ${ }^{4}$, Manuela Pennisi ${ }^{4}$, Rita Bella $^{1}$, Giuseppe Lanza $^{2,5, *}$

${ }^{1}$ Department of Medical and Surgical Sciences and Advanced Technologies, University of Catania, 95123 Catania, Italy

${ }^{2}$ Oasi Research Institute-IRCCS, 94018 Troina, Italy

${ }^{3}$ Ospedale Maggiore di Modica, ASP Ragusa, 97015 Modica, Italy

${ }^{4}$ Department of Biomedical and Biotechnological Sciences, University of Catania, 95123 Catania, Italy

${ }^{5}$ Department of Surgery and Medical-Surgery Specialties, University of Catania, 95123 Catania, Italy

*Correspondence: glanza@oasi.en.it (Giuseppe Lanza)

${ }^{\dagger}$ These authors contributed equally.

\section{DOI:10.31083/j.jin2101008}

This is an open access article under the CC BY 4.0 license (https://creativecommons.org/licenses/by/4.0/).

Submitted: 6 February 2021 Revised: 9 March 2021 Accepted: 7 May 2021 Published: 28 January 2022

We report on our remote speech therapy experience in post-stroke aphasia. The aim was to test the feasibility and utility of telerehabilitation to support future randomized controlled trials. Post-stroke aphasia is a common and disabling speech disorder, which significantly affects patients' and caregivers' health and quality of life. Due to COVID-19 pandemic, most of the conventional speech therapy approaches had to stop or "switch" into telerehabilitation procedures to ensure the safety of patients and operators but, concomitantly, the best rehabilitation level possible. Here, we planned a 5-month telespeech therapy programme, twice per week, of a patient with non-fluent aphasia following an intracerebral haemorrhage. Overall, treatment adherence based on the operator's assessments was high, and incomplete adherence for technical problems occurred very rarely. In line with the patient's feedback, acceptability was also positive, since he was constantly motivated during the sessions and the exercises performed autonomously, as confirmed by the speech therapist and caregiver, respectively. Moreover, despite the sequelae from the cerebrovascular event, evident in some writing tests due to the motor deficits in his right arm and the disadvantages typical of all telepractices, more relevant results were achieved during the telerehabilitation period compared to those of the "face-to-face" therapy before the COVID-19 outbreak. The telespeech therapy performed can be considered successful and the patient was able to return to work. Concluding, we support it as a feasible approach offering patients and their families the opportunity to continue the speech and language rehabilitation pathway, even at the time of pandemic.

Keywords

Aphasia; Telerehabilitation; COVID-19 pandemic; Stroke; Speech therapy

\section{Introduction}

Aphasia is a common acquired speech disorder, most often seen after stroke, affecting 30-40\% of acute stroke survivors [1]. Post-stroke aphasia (PSA) is extremely disabling and may show different degrees of impairment, from expressive lan- guage to verbal comprehension deficit, and from reading to writing disorders. PSA is also a key prognostic factor for functional outcome [2]. Most of the spontaneous recovery usually occurs within the first three months following the event; then, the recovery rate progressively slows down, and only little additional improvement can be expected after the first year [3, 4].

Speech therapy for PSA promotes the recovery of functional communication, receptive language, and expressive language, although the efficacy of treatment is lower in the chronic than in the acute phase [5]. Solid evidence supports the effectiveness of speech and language therapy on PSA, although providing the rehabilitation regimes described in the trial protocols in a local or clinical setting is often challenging [6]. This is also a consequence of the growing demand, increasing cost, constrained resources, and limited speech and language pathology (SLP) therapists trained in PSA, as well as of some geographical barriers and co-morbidities (e.g., motor deficit and fatigue) commonly seen in persons with stroke [7].

Recent studies propose telerehabilitation (TRH) for the assessment and treatment of aphasic patients. As part of telemedicine and telepractice, TRH offers remote services using telecommunications technologies (such as video, audio, and virtual reality), thus reducing hospitalization, costs, and barriers imposed by the distance. A recent review [8] supports the use of telepractice for adult patients with aphasia, although further studies are needed to demonstrate its efficacy. Notably, most studies are conducted in the patient's own home with a personal computer (PC)-based videoconferencing system [9], thus providing a feasible and easy-toaccess format $[6,10]$. More recently, preliminary evidence of the usefulness of a tablet-based platform to deliver tailored 
language and cognitive therapy to individuals with aphasia has been reported [11], as well as findings suggesting that self-delivered therapy (via App) is beneficial for chronic expressive aphasia [12]. Overall, aphasia therapy delivered by PC or tablets is a useful adjunct to standard practice, although some technical and procedural factors need to be considered before their systematic implementation [13].

In this scenario, because of the COronaVirus DIsease19 (COVID-19) pandemic [14, 15], during the last year most PSA have not been treated or have been treated partially/not properly, due to the social and geographical distancing adopted to prevent the infection [14, 16, 17]. More importantly, most healthcare services considered not urgent or elective (such as the SLP clinics) have experienced a considerable interruption or fragmentation of their activities [18]. Therefore, the COVID-19 pandemic has obliged the healthcare system to significantly transform its service offer and delivery modality, thus promoting a rapid expansion of the telehealth and TRH [19-21].

Here, we report a case of a patient with PSA at the time of COVID-19 pandemic, during which he received augmented telespeech therapy. The aim is to provide insights on the feasibility and utility to further support future large-scale randomized controlled trials.

\section{Case report}

\subsection{Clinical background}

A 54-year-old right-handed Caucasian man of Sicilian ancestry was admitted at the Oasi Research Institute-IRCCS of Troina (Italy), where he worked as Information and Communications Technology (ICT) manager. Both patient and caregiver (i.e., his wife) received detailed information. An informed consent form accessible to people with aphasia was used, and a written consent was obtained from the patient.

Cardiovascular disease, stroke, and dementia were reported in his family history, whereas past medical history included hypertension and dyslipidaemia. He was on daily treatment with pantoprazole, irbesartan, and simvastatin. Approximately one year earlier (July 2019), he suddenly presented with confusional state, non-fluent aphasia, and motor deficit of the right limbs. At the Emergency Department, the brain computed tomography (CT) scan showed an intracerebral haemorrhage of the left nuclear-capsular and frontal region. Because of the clinical and neuroradiological worsening, with evidence of an initial midline shifting of the left lateral ventricle, the patient was transferred to the Intensive Care Unit. A few days after, he underwent a left frontal minicraniotomy, hematoma drainage, and refixing of the bone operculum. Post-operative CT showed improvement of the affected areas, along with clinical recovery.

At the neurological examination, he was collaborating and partially oriented, and showed: global mainly non-fluent aphasia, right facial nerve central palsy, right hemiparesis, brisk tendon reflexes and sensory deficit at the right limbs, and right spastic gait. Routine laboratory exams were nor- mal, as well as thyroid hormones, coagulation, and Severe Acute Respiratory Syndrome-Coronavirus-2 (SARS-CoV-2) test. Electrocardiogram showed signs of hypertensive cardiopathy, whereas chest X-ray was unremarkable. Electroencephalogram revealed sub-continuous non-reactive thetadelta activity over the left frontal and temporal regions. After discharge, he underwent motor, speech, and cognitive rehabilitation. All data were obtained with the informed consent of the participant.

\subsection{Telespeech and language therapy}

The patient started a conventional SLP therapy, which, however, was early stopped due to the pandemic. The baseline assessment through the standardized comprehensive test for Italian aphasic patients, called ENPA ("Esame Neuropsicologico per l'Afasia", that is "Neuropsychological Exam for Aphasia”) [22], showed non-fluent aphasia, anomies, circumlocutions, phonemic paraphasia, and deficit of narration, morphosyntax, pragmatic skills, and working memory. He also showed an impairment of writing (even for dictated words), comprehension, and word generation. Finally, when asked to repeat, he was unable without significant difficulties and repeated attempts to self-correct himself (the so-called "conduites d'approche").

Remote SLP therapy was initiated, by using the wellknown free platform "Skype", from the operators' study laptop to the patient's home laptop, which were used for all the sessions. The technical setup included an external speaker to improve sound quality and a wide-angle web camera to enable also review of body language and other gestures. Patient and caregiver were given a short training on the use of the software before starting the TRH programme. All sessions were provided through a stable broadband connection.

A senior SLP therapist (F.S.), expert in PSA, delivered the TRH, assisted by an undergraduate SLP therapist (L.C.). All the interventions were tailored to the patient's impairment and needs, and used a comprehensive approach to assess and enhance all aspects of language. The goal was to facilitate the access to the lexical store, to improve oral and written understanding, to strengthen narrative skills and working memory, to widen sentence length, and to stimulate pragmatic and narrative skills. They also used collections of tasks comprising individual aphasia exercises, for instance, oral and written naming and reading sentences and text. Furthermore, maps, pictures, and other items were used as additional resources.

Timing and duration of each session were established by the SLP therapist and arranged with the patient and his caregiver, according to the Template for Intervention Description and Replication (TIDieR) checklist for patients with PSA [23]. An intervention based on a duration of two hours and a frequency of twice per week was delivered, resulting in an intensity of 4 hours of therapy per week and a total dosage therapy of 88 hours planned. In some cases, due to some technical inconvenience, need for more breaks, explicit request of the patient, or recovery of time lost for a previously skipped session (which, however, occurred only twice, not 
consecutively), a longer time (up to 2.5 hours) was delivered to adjust to the participant's timetable and other planned activities, but only if he was able to withstand a longer session. Sessions were delivered over five consecutive months, from March to July 2020. Logistic and/or technical issues occurred very rarely (none of the sessions was stopped, slowed down, or disturbed due to low Internet connection), as well as no adverse event or medical complication during the abovementioned period.

At the end of the TRH, the patient was reassessed by means of the same tests used at baseline (ENPA) [22]. Given that the baseline evaluation was carried out in a "face-to-face" modality, also the post therapy assessment took place in person. This allowed a more reproducible comparison and made the writing parts of the test more reliable than an on-line remote examination. He improved in the following skills: repetition of non-words, writing of words, reading of sentences, oral naming of verbs, written naming of verbs and names, oral and visual comprehension of words; the generation of words list, which was severely affected at baseline, improved substantially. Conversely, other abilities (i.e., repetition of words and sentences, writing of non-words and sentences, reading of words and non-words, oral naming of names and colours, oral and visual comprehension of sentences) did not vary or did not further improve following the TRH. After the end of the lockdown period, the patient continued his rehabilitation program in a conventional "face-to-face" modality only. The choice to give TRH in addition to the usual care is still a debated issue, as it challenges the possibility to determine the effects of each rehabilitation modality.

Table 1 (Ref. [23]) summarizes all the participant's and SLP intervention items and data, based on the TIDieR checklist [23].

\section{Discussion \\ 3.1 Main findings}

The COVID-19 pandemic represented a challenge for adaptation on several fronts. Studies on teletherapy during the pandemic [24] have shown that COVID-19 is broadly impacting neurorehabilitation, including SLP services: referral patterns are less numerous and different, usual care has been disrupted and interventions have been modified, eventually affecting the impact on outcomes. However, the need for social distancing and the extreme care to reduce the spread of infection anticipated changes that were actually already possible, such as those regarding the use of telehealth. In particular, telemedicine and technology can be used to upgrade rehabilitative care, especially within the setting of shorter hospitalization stays, social distancing and confinement, and limited access and resources [25], as recently highlighted by the application of lexical retrieval training in tablet-based speechlanguage rehabilitation of aphasia [26]. For patients who received SLP therapy, it was found that the outcomes did not change significantly [24].
In this case, a TRH-based approach has proven to be a feasible tool to overcome the limits imposed by the lockdown, especially in terms of healthcare reorganization, and to support the role of technology and as a strong ally during the pandemic. Overall, treatment adherence based on the operator's assessments was high, and incomplete adherence for technical problems occurred very rarely. Due to the motor deficit in his right side, the adherence to some writing tasks was not always optimal; however, he managed to perform the tasks requested and to show the results to the operators with the assistance of his caregiver. In line with the patient's feedback, acceptability of the telespeech therapy was also positive, since he was constantly motivated during the sessions and the exercises performed autonomously, as confirmed by the SLP therapist and patient's caregiver, respectively.

The implementation of telespeech therapy has recently been described [27]. The purpose is to guarantee the continuity of care services for patients who could worsen during the period of social distancing. Consultations, carried out through synchronous video calls by all participants (patients, caregivers, SLP therapists, and students), enabled a remote care with the same quality of "face-to-face" interventions. In this context, it cannot be excluded that telehealth technologies will be part of the professional reality in the near future, both to respond to the needs imposed by the pandemic and to expand the offer of speech therapy services worldwide [28].

Telepractice also facilitates healthcare services in exceptional settings and situations, being the ongoing pandemic one of these. A recent online survey [29] has shown that both patients and operators were satisfied because telepractice allowed them to receive and provide, respectively, clinical services in an exceptional scenario. The main reasons for client refusal or discontinuation were the lack of proper equipment, insufficient independence, and doubts on the effectiveness of such a modality [29]. This is in line with the fact that while COVID-19 has propelled the use of telemedicine to the forefront, many previous barriers to telemedicine implementation still exist. These include the actual efficacy of an "urgent telemedicine" (especially in terms of feasibility, patient satisfaction, and outcomes), payment and reimbursement policies, administrative regulations, cyber and ethical security, and technology education and utilization, among others [30].

Notwithstanding these issues, TRH for stroke survivors has emerged as a promising intervention for motor, speech, cognitive, occupational, and other therapies. A recent systematic review of 22 publications suggested that TRH has either better or equal effects on motor recovery, higher cortical deficits, and mood disorders compared with conventional "face-to-face" therapy [31]. However, current evidence in PSA is still preliminary, with only few videoconference trials [32]. A recent randomized controlled pilot study in 30 PSA patients showed that synchronous TRH was safe and acceptable, with tolerable technical issues and high satisfaction among both patients and operators [33]. However, complex $\mathrm{TRH}$ interventions require a higher degree of technical ex- 
Table 1. Participant's and speech and language pathology intervention items and data, based on the Template for Intervention Description and Replication (TIDieR) checklist [23].

\begin{tabular}{|c|c|}
\hline \multicolumn{2}{|l|}{ Participant } \\
\hline Demographics & $\begin{array}{l}\text { 54-year-old, right-handed, Caucasian, Italian-speaking man; normal psychomotor development and premorbid cogni- } \\
\text { tive status; high-school diploma; former Information and Communications Technology (ICT) manager }\end{array}$ \\
\hline Stroke & $\begin{array}{l}\text { July 2019; baseline NIHSS: 9; left nuclear-capsular and frontal hemorrhagic stroke; no prior stroke, normal mental } \\
\text { health and cognition prior to stroke; mild vascular cognitive impairment following stroke; no preexisting neurological } \\
\text { diagnosis, no visual or hearing impairment; hypertension and dyslipidaemia }\end{array}$ \\
\hline Environmental details & $\begin{array}{l}\text { Adequate living context prior to stroke; supportive living context following stroke; good social support (wife) and socio- } \\
\text { economic status }\end{array}$ \\
\hline \multicolumn{2}{|c|}{ Speech and language pathology } \\
\hline Why & $\begin{array}{l}\text { To recover the fluency through rehabilitation and training of all cognitive and semantic aspects of language. Goals: to } \\
\text { facilitate the access to the lexical storage, to improve oral and written understanding, to strengthen narrative skills and } \\
\text { working memory, to increase sentence length, and to stimulate pragmatic and narrative skills }\end{array}$ \\
\hline What was delivered & $\begin{array}{l}\text { Collection of tasks comprising aphasia exercises (oral and written naming and reading sentences and text), maps, pic- } \\
\text { tures, and other additional resources in some sessions. The exercises were based on lexical evocation with the use of } \\
\text { a dictionary, exercises of lexical decision-making, explanation of homograph words, tests on synonyms and opposites, } \\
\text { target word completion from a single phoneme, completion of a target word with missing phoneme, explanation of } \\
\text { the meaning of ambiguous sentences, exercises on semantic categories (categorization, identification of the intruder be- } \\
\text { tween four terms, etc.), identification of the word starting from the definition, reading and narration through conceptual } \\
\text { maps, writing and reading of news report, explanation of proverbs, and free conversation }\end{array}$ \\
\hline Who provided & $\begin{array}{l}\text { A senior SLP therapist, expert in post-stroke aphasia, delivered all sessions, assisted by an undergraduate SLP therapist } \\
\text { in training }\end{array}$ \\
\hline How was therapy provided & $\begin{array}{l}\text { The patient started a "face-to-face", "1-to-1", conventional SLP therapy which was stopped early, due to the COVID-19 } \\
\text { pandemic. A computer-based telerehabilitation was then initiated through the free platform "Skype", in synchronous } \\
\text { audio/video phone call }\end{array}$ \\
\hline Where & $\begin{array}{l}\text { Conventional therapy was performed in a dedicated out-patient Clinic for speech and language pathology of a regional } \\
\text { clinical-research Center dealing with neurological disorders. Remote therapy took place from the operator's office to } \\
\text { the patient's home }\end{array}$ \\
\hline How much & $\begin{array}{l}\text { Two hours per session, twice per week, resulting in an intensity of } 4 \text { hours per week and a total therapy dosage of } 88 \\
\text { hours planned }\end{array}$ \\
\hline Tailoring & Conventional and remote therapies were tailored both on the patient's difficulty and functional relevance \\
\hline Modifications & $\begin{array}{l}\text { The patient experienced no specific difficulty and totally agreed with the telerehabilitation-based approach, showing a } \\
\text { high and constant level of acceptance and compliance. Moreover, as a former ICT manager, he was already skilled with } \\
\text { the use of the computer and the main applications for video chat and voice calls, such as Skype. Therefore, he had no } \\
\text { difficulty in accessing Skype, even during training, and no specific therapy or protocol modification/adjustments were } \\
\text { needed for the translation from conventional to remote sessions }\end{array}$ \\
\hline Adherence & $\begin{array}{l}\text { The patient showed an overall good adherence to both clinics-based interventions and home practice tasks. Due to the } \\
\text { motor deficit in his right side, the adherence to some writing tasks was not always optimal; however, he managed to } \\
\text { perform the tasks requested and to show the results to the operator with the assistance of his caregiver }\end{array}$ \\
\hline
\end{tabular}

ICT, Information and Communications Technology; NIHSS, National Institutes of Health Stroke Scale; SLP, speech and language pathology.

pertise and long-term effects are still unclear. In particular, there are still few trials exploring how TRH can be used to increase therapy time and the impact that such augmented intervention might have on the outcome measures [34, 35].

In the patient with PSA described here, although some skills did not improve, the most important goals could be considered achieved, leading to a global increase in his language abilities. The effects of treatment were subjectively evident already during the interventions and were confirmed at the end of the TRH, thus suggesting that this approach may have influenced the patient's skills beyond a single speech ability [10]. It is also possible that tailoring the intervention to the baseline patient's impairment might have endorsed a greater generalization of speech-related skills. These results are also in line with the overall effects shown by videoconferencebased TRH in brain-injured subjects, including stroke, and have translational implications for further remote applications [36].

As known, the most powerful predictor of PSA recovery is the initial severity of aphasia, given that greater severity is associated with poorer improvement [37]. However, this report suggests that improvement may persist over time and depend on the usage time [6]. A previous study reported that also the number of hours per week of TRH correlated with recovery and that at least 4 hours per week (as carried out in the present case) or 24 hours in four weeks were 
required to show significant results [38]. Nevertheless, although PC, tablets, and other devices have great potential for personalized home practice to maintain and augment traditional aphasia rehabilitation, both attitude and motivation to use the technology, as well as an adequate training, seem to be even more important factors than age and aphasia type or severity [39]. As a former ICT manager, the patient reported here was already skilled with the use of the PC and the main telecommunication applications specialized in video chat and voice calls. As such, he did not have any difficulty in accessing Skype, even during training, and no specific therapy or protocol modification/adjustments were needed when translating from conventional to remote sessions.

Lastly, we confirmed the helpful guidance of caregivers (i.e., the patient's wife in this report), since they usually assisted the patient in the management of technology and home exercises [40]. In the pandemic scenario, social distance provides more family time, and in some cases, better organization for carrying out the exercises prescribed, with a positive impact on the user prognosis [27]. In other words, as Volkers stated, "family members develop activities previously performed by therapists" [41].

\subsection{Strengths and limitations}

The main contribution of this case is the use of a videoconference tool for TRH, including the description of the patient's acceptance, compliance, and adherence with the method. Overall, this approach has shown positive results, probably due to the delivery of the therapy in a more comfortable environment and through a previously known communication platform.

Other strengths of TRH are the possibility to fully use the time available, to interact more with the caregiver, to provide therapy directly in the patient's living environment, and to arrange the sessions easily. Home-based therapy for aphasic patients has proven to be as effective as in-clinic session, with the added advantage that patients can practice more and in a more comfortable way [42]. Increased autonomy and opportunity for practice with the remotely monitored therapy has also been acknowledged, but the supervision of a trained SLP therapist (as that engaged in the present report) remains crucial [43]

The main limitation of this report is that it is based on a single case, with pre- and post-language test performed by the same operator. Another caveat it that evidence of generalizability to other clinical contexts or individuals with other types of aphasia or speech disorders (such as progressive aphasia) cannot be provided.

Third, although we have found effects in some language abilities, but not all, these might have been due to the increase in the total therapy time received. On the other hand, it might also due to the fact that, to date, only few SLP therapists have received an adequate training for switching from an in-person service to a synchronous telepractice model. This new and unexpected environment shift may have altered vocal behaviours and communication interactions, thus neg- atively impacting some treatment outcomes [44]. A sudden change from in-person to online clinical services, such as that imposed by the COVID-19, requires novel strategies and specific support to address specific pandemic-related issues.

Lastly, other disadvantages of TRH are the same of telemedicine, i.e., the lack of a direct contact between patient and therapist; the preliminary adaptation of both user and therapist, often requiring additional consultation time and more session duration; less socialization, which is instead a relevant component of the in-hospital rehabilitation; the need that all patient's rights are maintained by efficient and confidential service [45]; slow Internet connection and other technical issues (e.g., low quality of sound and/or video, etc.) [46].

\section{Conclusions}

We provided our TRH service during the COVID-19 pandemic to a patient with PSA. Although each individual case must be assessed for eligibility, telespeech therapy might provide the necessary healthcare assistance in case of social distancing, such as that imposed by the COVID-19. Notwithstanding the limitations of a case report, the SLP therapy can be considered successful and the patient was able to return to work and carry out autonomously his previous activities. Therefore, we support it as a feasible approach offering patients and their families the opportunity to continue the speech and language rehabilitation pathway, even at the time of a pandemic. Randomized controlled trials with follow-up are needed to confirm the utility and verify the effectiveness of telespeech therapy in PSA and other clinical contexts.

\section{Abbreviations}

COVID-19, COronaVirus DIsease-19; CT, computed tomography; ENPA, Esame Neuropsicologico per l'Afasia; ICT, Information and Communications Technology; PC, personal computer; PSA, post-stroke aphasia; SARS-CoV-2, Severe Acute Respiratory Syndrome-Coronavirus-2; SLP, speech and language pathology; TIDieR, Template for Intervention Description and Replication; TRH, telerehabilitation.

\section{Author contributions}

LC, FS, and GL designed the overall concept and outline of the manuscript; DG, MT, MPe and DM contributed to the discussion and design of the manuscript; SP, MPa, VN, and FF contributed to the writing and editing the manuscript and illustrations; FIIC, RF, SF, and RB reviewed the literature and the final draft of the manuscript. All authors have read and agreed to the published version of the manuscript.

\section{Ethics approval and consent to participate}

All procedures were carried out in accordance with the recommendations of the Declaration of Helsinki and its later amendments. All data were obtained with the informed consent of the participant. 


\section{Acknowledgment}

We would like to thank very much the patient and his wife.

\section{Funding}

This research received no external funding.

\section{Conflict of interest}

The authors declare no conflict of interest.

\section{References}

[1] Flowers HL, Skoretz SA, Silver FL, Rochon E, Fang J, FlamandRoze C, et al. Poststroke Aphasia Frequency, Recovery, and Outcomes: a Systematic Review and Meta-Analysis. Archives of Physical Medicine and Rehabilitation. 2016; 97: 2188-2201.e8.

[2] Gialanella B. Aphasia assessment and functional outcome prediction in patients with aphasia after stroke. Journal of Neurology. 2011; 258: 343-349.

[3] Ferro JM, Mariano G, Madureira S. Recovery from aphasia and neglect. Cerebrovascular Diseases. 1999; 9: 6-22.

[4] Fisicaro F, Lanza G, Grasso AA, Pennisi G, Bella R, Paulus W, et al. Repetitive transcranial magnetic stimulation in stroke rehabilitation: review of the current evidence and pitfalls. Therapeutic Advances in Neurological Disorders. 2019; 12: 1-22.

[5] Brady MC, Kelly H, Godwin J, Enderby P. Speech and language therapy for aphasia following stroke. Cochrane Database of Systematic Reviews. 2012; 16: CD000425.

[6] Bhogal SK, Teasell R, Speechley M. Intensity of aphasia therapy, impact on recovery. Stroke. 2003; 34: 987-993.

[7] Theodoros DG. Telerehabilitation for service delivery in speechlanguage pathology. Journal of Telemedicine and Telecare. 2008; 14: $221-224$.

[8] Weidner K, Lowman J. Telepractice for Adult Speech-Language Pathology Services: a Systematic Review. Perspectives of the ASHA Special Interest Groups. 2020; 5: 326-338.

[9] Cherney LR, van Vuuren S. Telerehabilitation, virtual therapists, and acquired neurologic speech and language disorders. Seminars in Speech and Language. 2012; 33: 243-257.

[10] Øra HP, Kirmess M, Brady MC, Partee I, Hognestad RB, Johannessen $\mathrm{BB}$, et al. The effect of augmented speech-language therapy delivered by telerehabilitation on poststroke aphasia-a pilot randomized controlled trial. Clinical Rehabilitation. 2020; 34: 369 381.

[11] Des Roches CA, Balachandran I, Ascenso EM, Tripodis Y, Kiran S. Effectiveness of an impairment-based individualized rehabilitation program using an iPad-based software platform. Frontiers in Human Neuroscience. 2015; 8: 1015.

[12] Stark BC, Warburton EA. Improved language in chronic aphasia after self-delivered iPad speech therapy. Neuropsychological Rehabilitation. 2018; 28: 818-831.

[13] Finch E, Clark K, Hill A. Using computer-based therapy as an adjunct to standard anomia therapy. Journal of Clinical Practice in Speech-Language Pathology. 2013; 15: 2-6.

[14] Tsatsakis A, Calina D, Falzone L, Petrakis D, Mitrut R, Siokas V, et al. SARS-CoV-2 pathophysiology and its clinical implications: an integrative overview of the pharmacotherapeutic management of COVID-19. Food and Chemical Toxicology. 2020; 146: 111769.

[15] Fisicaro F, Di Napoli M, Liberto A, Fanella M, Di Stasio F, Pennisi M, et al. Neurological Sequelae in Patients with COVID-19: A Histopathological Perspective. International Journal of Environmental Research and Public Health. 2021; 18: 1415.

[16] Pennisi M, Lanza G, Falzone L, Fisicaro F, Ferri R, Bella R. SARSCoV-2 and the Nervous System: From Clinical Features to Molecular Mechanisms. International Journal of Molecular Sciences. 2020; 21: 5475 .

[17] Maniaci A, Iannella G, Vicini C, Pavone P, Nunnari G, Falsaperla
$\mathrm{R}$, et al. A case of covid-19 with late-onset rash and transient loss of taste and smell in a 15-year-old boy. American Journal of Case Reports. 2020; 21: e925813.

[18] Wosik J, Fudim M, Cameron B, Gellad ZF, Cho A, Phinney D, et al. Telehealth transformation: COVID-19 and the rise of virtual care. Journal of the American Medical Informatics Association. 2020; 27: 957-962.

[19] Tenforde AS, Borgstrom H, Polich G, Steere H, Davis IS, Cotton $\mathrm{K}$, et al. Outpatient Physical, Occupational, and Speech Therapy Synchronous Telemedicine: A Survey Study of Patient Satisfaction with Virtual Visits During the COVID-19 Pandemic. American Journal of Physical Medicine \& Rehabilitation. 2020; 99: 977981.

[20] Cocuzza S, Maniaci A, Grillo C, Ferlito S, Spinato G, Coco S, et al. Voice-Related Quality of Life in Post-Laryngectomy Rehabilitation: Tracheoesophageal Fistula's Wellness. International Journal of Environmental Research and Public Health. 2020; 17: 4605.

[21] Ferlito S, Maniaci A, Di Luca M, Grillo C, Mannelli L, Salvatore $\mathrm{M}$, et al. From Uncommon Infection to Multi-Cranial Palsy: Malignant External Otitis Insights. Dose-Response. 2020; 18: 155932582096391.

[22] Capasso R, Miceli G. Esame Neuropsicologico per l'Afasia: E.N.P.A. Springer-Verlag Mailand: Milan. 2001.

[23] RELEASE Collaboration. Communicating simply, but not too simply: Reporting of participants and speech and language interventions for aphasia after stroke. International Journal of SpeechLanguage Pathology. 2020; 22: 302-312.

[24] Chadd K, Moyse K, Enderby P. Impact of COVID-19 on the Speech and Language Therapy Profession and Their Patients. Frontiers in Neurology. 2021; 12: 629190.

[25] Rutledge CM, Haney T, Bordelon M, Renaud M, Fowler C. Telehealth: preparing advanced practice nurses to address healthcare needs in rural and underserved populations. International Journal of Nursing Education Scholarship. 2014; 11: /j/ijnes.2014.11.issue-1/ijnes-2013-0061/ijnes-2013-0061.xml.

[26] Gallée J, Pittmann R, Pennington S, Vallila-Rohter S. The Application of Lexical Retrieval Training in Tablet-Based SpeechLanguage Intervention. Frontiers in Neurology. 2020; 11: 583246.

[27] Dimer NA, Canto-Soares ND, Santos-Teixeira LD, Goulart BNG The COVID-19 pandemic and the implementation of telehealth in speech-language and hearing therapy for patients at home: an experience report. CoDAS. 2020; 32: e20200144.

[28] Fernandes FDM, Lopes-Herrera SA, Perissinoto J, MoliniAvejonas DR, Higuera Amato CA, Tamanaha AC, et al. Use of telehealth by undergraduate students in Speech Therapy: possibilities and perspectives during COVID-19 pandemic. CoDAS. 2020; 32 : e20200190.

[29] Kraljević JK, Matić A, Dokoza KP. Telepractice as a Reaction to the COVID-19 Crisis: Insights from Croatian SLP Settings. International Journal of Telerehabilitation. 2020; 12: 93-104.

[30] Zughni LA, Gillespie AI, Hatcher JL, Rubin AD, Giliberto JP. Telemedicine and the Interdisciplinary Clinic Model: during the COVID-19 Pandemic and beyond. Otolaryngology-Head and Neck Surgery. 2020; 163: 673-675.

[31] Sarfo FS, Ulasavets U, Opare-Sem OK, Ovbiagele B. TeleRehabilitation after Stroke: an Updated Systematic Review of the Literature. Journal of Stroke and Cerebrovascular Diseases. 2018; 27: 2306-2318.

[32] Øra HP, Kirmess M, Brady MC, Winsnes IE, Hansen SM, Becker F. Telerehabilitation for aphasia - protocol of a pragmatic, exploratory, pilot randomized controlled trial. Trials. 2018; 19: 208.

[33] Øra HP, Kirmess M, Brady MC, Sørli H, Becker F. Technical Features, Feasibility, and Acceptability of Augmented Telerehabilitation in Post-stroke Aphasia-Experiences From a Randomized Controlled Trial. Frontiers in Neurology. 2020; 11: 671.

[34] Mashima PA, Doarn CR. Overview of telehealth activities in speech-language pathology. Telemedicine Journal and E-Health. 2008; 14: 1101-1117.

[35] Hall N, Boisvert M, Steele R. Telepractice in the assessment and 
treatment of individuals with aphasia: a systematic review. International Journal of Telerehabilitation. 2013; 5: 27-38.

[36] Brennan DM, Georgeadis AC, Baron CR, Barker LM. The effect of videoconference-based telerehabilitation on story retelling performance by brain-injured subjects and its implications for remote speech-language therapy. Telemedicine Journal and EHealth. 2004; 10: 147-154.

[37] Pedersen PM, Vinter K, Olsen TS. Aphasia after stroke: type, severity and prognosis. the Copenhagen aphasia study. Cerebrovascular Diseases. 2004; 17: 35-43.

[38] Meyer AM, Getz HR, Brennan DM, Hu TM, Friedman RB. Telerehabilitation of Anomia in Primary Progressive Aphasia. Aphasiology. 2016; 30: 483-507.

[39] Kurland J, Wilkins AR, Stokes P. IPractice: piloting the effectiveness of a tablet-based home practice program in aphasia treatment. Seminars in Speech and Language. 2014; 35: 51-63.

[40] Boldrini P, Kiekens C, Bargellesi S, Brianti R, Galeri S, Lucca L, et al. First impact of COVID-19 on services and their preparation. European Journal of Physical and Rehabilitation Medicine. 2020; 56: $319-322$.
[41] Volkers N. Work: only its Name will Stay the same. The ASHA Leader. 2019; 24: 42-51.

[42] Godlove J, Kiran S. Is Home Based Therapy as Effective in-Clinic Therapy for Patients with Aphasia? Archives of Physical Medicine and Rehabilitation. 2017; 98: e77-e78.

[43] Wade J, Mortley J, Enderby P. Talk about it: Views of people with aphasia and their partners on receiving remotely monitored computer-based word finding therapy. Aphasiology. 2003; 17: 10311056.

[44] Knickerbocker K, Bryan C, Ziegler A. Phonogenic Voice Problems among Speech-Language Pathologists in Synchronous Telepractice: an Overview and Recommendations. Seminars in Speech and Language. 2021; 42: 73-84.

[45] Chaet D, Clearfield R, Sabin JE, Skimming K. Ethical practice in Telehealth and Telemedicine. Journal of General Internal Medicine. 2017; 32: 1136-1140.

[46] Almathami HKY, Win KT, Vlahu-Gjorgievska E. Barriers and Facilitators That Influence Telemedicine-Based, Real-Time, Online Consultation at Patients' Homes: Systematic Literature Review. Journal of Medical Internet Research. 2020; 22: e16407. 